\title{
Optimización de una Célda Solar de GaAs/AIGaAs
}

\author{
Cristhian Manuel Duran Acevedo
}

Departamento de Electrónica, Eléctrica, Sistemas y Telecomunicaciones .Facultad de Ingenierías y Arquitectura. Grupo GISM, Universidad de Pamplona

\section{Resumen}

El presente artículo consiste en la optimización de una celda solar fotovoltaica de material de GaAs/AIGaAs, usando el programa de diseño y simulación PC1D. Mediante el programa de simulación se visualizan los resultados gráficos. Se presentan las respuestas a ciertos parámetros que son fundamentales para determinar la eficiencia del material y el comportamiento a ciertos factores externos, como el tipo de excitación a la que el dispositivo es sometido. A partir de esto se obtienen sus principales características: Corriente de cortocircuito Isc, Tensión de circuito abierto Voc, Eficiencia cuántica QE, y Factor de llenado FF. Como resultado del estudio se obtuvo una eficiencia del $70 \%$ a partir de un $6 \%$ con diferente longitud de onda, el cual indica un buen rendimiento de la celda solar.

Palabras claves: GaAs/AIGaAs; PC1D; Celda solar; Eficiencia; programa

\section{Optimization of a solar Cell of GaAs/AIGaAs}

\section{Abstract}

The present article consists in the optimization of a solar photovoltaic cell of GaAs / AIGaAs material, using the design and simulation program PC1D.

The graphic results are displayed through the simulation program. We present the answers to certain parameters that are fundamental to determine the efficiency of the material and the behavior to certain external factors, such as the type of excitation to which the device is subjected. From this, its main characteristics are obtained: Short circuit current Isc, Open circuit voltage Voc, Quantum efficiency QE, and Filling factor FF. As a result of the study, an efficiency of $70 \%$ was obtained from $6 \%$ with different wavelength, which indicates a good performance of the solar cell.

Keywords: GaAs/AIGaAs; PC1D; solar cell; Efficiency; Software 


\section{INTRODUCCION}

La célula o celda solar fotovoltaica es un dispositivo formado por una unión p- que transforma la energía luminosa del sol en energía eléctrica. Dicha conversión se lleva acabo de una manera limpia (es decir no se produce ningún tipo de contaminación debido al proceso de transformación). Estos dispositivos se fabrican usando materiales semiconductores como el Silicio (Si), el Arseniuro de Galio (GaAs), el Fosfuro de Indio (InP) entre otros.

Existe una gran variedad de células solares fabricadas con silicio monocristalino, con silicio policristalino, con material amorfo (silicio amorfo hidrogenado) o con diferentes materiales semiconductores (heterouniones).

Estos tipos responden a las diferentes necesidades del usuario, por ejemplo para uso doméstico, sistemas de bombeo y telefonía rural se hace uso extenso del silicio. Por otro lado, para usos en el espacio exterior algunos sistemas usan GaAs debido a que pueden soportar fuerte radiación solar. Uno de los parámetros fundamentales que caracterizan a las celdas solares es su eficiencia de conversión.

En esta revisión sobre la optimización de una celda solar se utiliza la herramienta PC1D la cual es un programa informático de simulación de celdas solares y que resuelve las ecuaciones no lineales totalmente acopladas para el transporte casi unidimensional de electrones y agujeros en dispositivos semiconductores cristalinos, con énfasis en dispositivos fotovoltaicos
(Hauga et al 2013; Cai 2018; Jiang et al 2011; Hussain et al 2015).

\section{Fundamentos teóricos}

Cuando la luz incide sobre un semiconductor Fig. 1 ( $\mathrm{Si}$ o GaAs), se crean pares de electrón - hueco en la región de agotamiento [6-7], una vez generados son barridos por un campo eléctrico existente en la región, de este modo los electrones son arrastrados dentro de la región $\mathrm{n}$ y los huecos a la región $p$.

En ausencia de un campo eléctrico, los electrones y los huecos se recombinaran entre si, y no se generara una señal detectable.

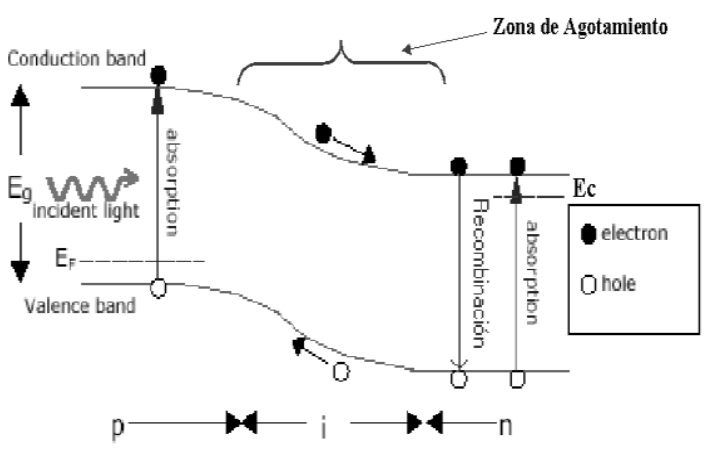

Fig.1. Diagrama de Bandas de una célula solar p-n. bajo iluminación.

Cabe señalar que no todos los fotones que llegan a la célula son absorbidos por ella, solamente lo harán los que tienen una energía igual o mayor que el ancho de banda prohibida del material semiconductor (Singh 2001). 
$h v \geq E_{g}$

Donde $\mathrm{h}$ es la constante de Planck, $\mathrm{v}$ es la frecuencia del fotón y $\mathrm{Eg}$ es la banda prohibida.

Estos fotones serán los que formarán los pares de electrón - hueco y debido al campo eléctrico producido por la unión de materiales en la célula de tipo $\mathrm{p}$ y $\mathrm{n}$ se separan antes de poder recombinarse, formándose así la corriente eléctrica que circula por la célula y su carga. Algunos fotones pueden no ser aprovechados para la creación de energía eléctrica por diferentes razones:

- Los fotones que tienen energía inferior al ancho de banda del semiconductor atraviesan el semiconductor sin ceder su energía para crear pares electrón hueco.

- Aunque un fotón tenga una energía mayor o igual al ancho de banda prohibida puede no ser aprovechado ya que una célula no tiene la capacidad de absorberlos a todos.

- Además, los fotones pueden ser reflejados en la superficie de la célula.

- Su principio de funcionamiento se basa en la capacidad de que los fotones de la radiación solar puedan transmitir su energía a los electrones de valencia de los materiales semiconductores, de manera que estos electrones
- rompen su enlace que anteriormente los tenía ligado a un átomo. Por cada enlace que se rompe queda un electrón y un hueco (falta de electrón en un enlace) para circular dentro del semiconductor.

- El movimiento de los electrones y huecos en sentidos opuestos (conseguido con la aplicación de un campo eléctrico), genera una corriente eléctrica en el semiconductor la cual puede circular por un circuito externo y liberar la energía cedida por los fotones para crear los pares electrón - hueco.

- El campo eléctrico necesario para la creación de la corriente eléctrica se consigue con la unión de dos semiconductores de diferente dopado: Un semiconductor tipo $p$ (exceso de huecos) y otro tipo $n$ (exceso de electrones). Al unirlos es creado el campo eléctrico.

- La cara iluminada será la de conductor tipo n y la no iluminada la de tipo $p$. Después se ubicaran los contactos, de manera que la cara no iluminada será cubierta totalmente por el contacto eléctrico (para ofrecer menos resistencia al paso de la corriente eléctrica) y la cara iluminada debe llevar un contacto por una parte lo más extenso posible, para ofrecer menos resistencia y por otra lo menos extenso posible para dejar pasar mayor cantidad de fotones. Lo más común es el uso de 
contactos en forma de peine (Esta forma deja pasar mayor número de fotones $y$ ofrece menos resistencia), Fig. 2.

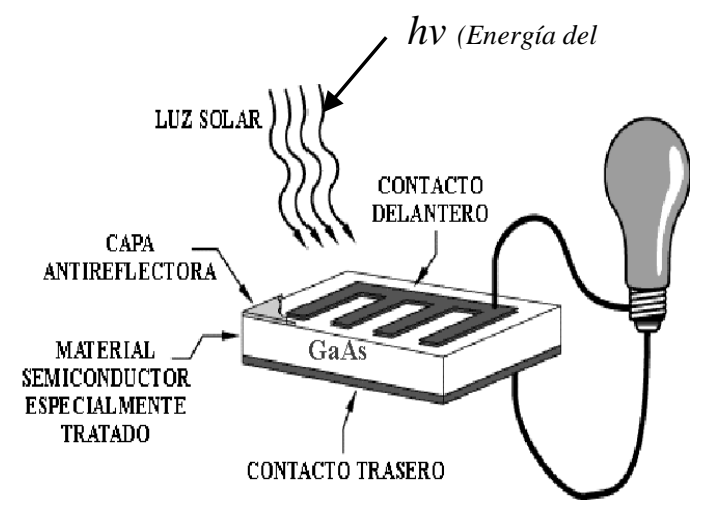

Fig.2. Estructura de una celular con carga y electrodos en forma de peine (Finger).

La capa antireflectora permite que los fotones no se pierdan antes de llegar a formar un par electrón - hueco. Los electrones libres son capturados y se genera una corriente eléctrica.

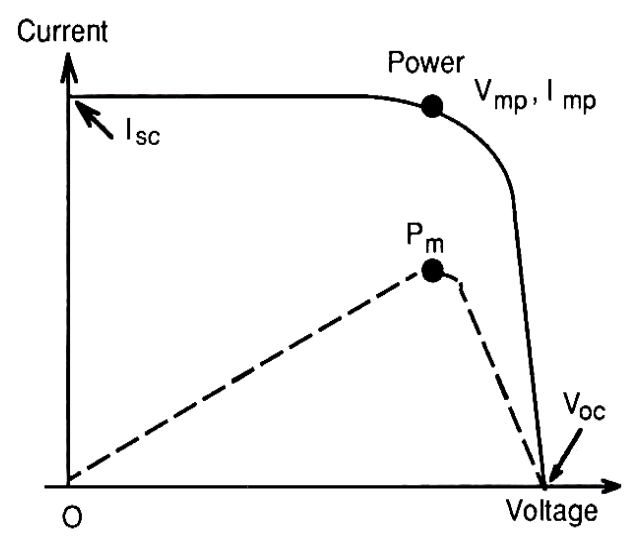

Fig.3. Representación típica de una curva de (I-V), se presenta la corriente de corto circuito $\left(\mathrm{Isc}_{\mathrm{sc}}\right)$, el Voltaje de circuito abierto $\left(V_{o c}\right)$ y el punto de máxima potencia $\left(V_{\mathrm{m}}, I_{\mathrm{mp}}\right)$.

\section{Parámetros importantes en las células solares}

En el cálculo de los parámetros (Liou 1994; Wenham et al 2011), se obtiene la potencia de una célula solar y se considera el caso en donde el diodo es empleado en modo de circuito abierto, y en la ecuación (2), bajo iluminación se representa los valores de (I-V) en que puede trabajar una célula fotovoltaica (Fig. 3).

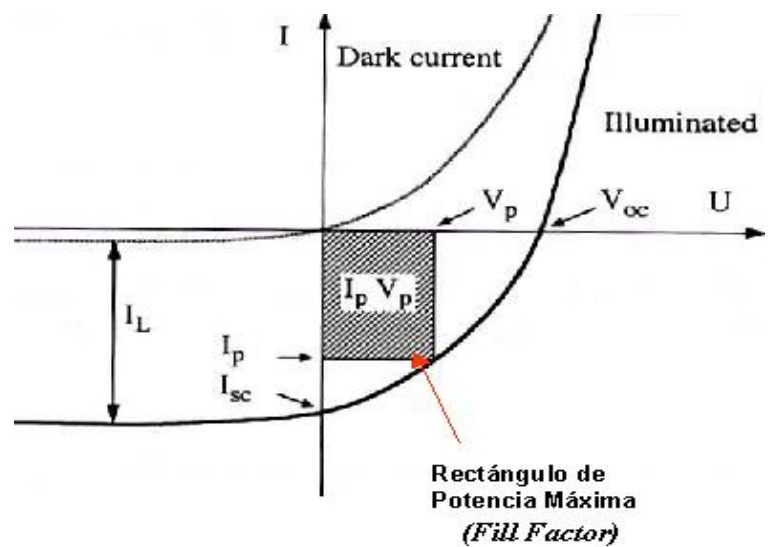

Fig.4. Características de I-V de una unión $p-n$, con corrientes en iluminación y en oscuridad.

El Fill Factor es el rectángulo de máxima potencia. La corriente entregada a una carga (IL) por una célula solar es debida a dos importantes factores (Fig. 4).

Corriente de iluminación: Debida a la generación de portadores que produce la iluminación.

Corriente de oscuridad: Debida a la recombinación de portadores que produce el voltaje externo necesario, para poder entregar energía a la carga. 
Bistua:Revista de la Facultad de Ciencias Básicas.2018.16(1):145-155

149

\section{A) Intensidad de corriente de cortocircuito (Isc)}

Definido como el máximo valor de corriente que circula por una célula fotovoltaica y se da cuando la célula está en cortocircuito $(\mathrm{V}=0)$.

$$
I=I_{L}-I_{S}\left[\exp \left(\frac{q V}{n K \quad T} \begin{array}{c}
B
\end{array}\right)-1\right]
$$

Donde:

V: Es la tensión de circuito abierto

q : Es la carga del electrón.

n: Factor de idealidad

KB: Es la constante de Boltzman

$\mathbf{T}$ : Temperatura en ${ }^{\circ} \mathrm{K}$

IL: Es la Fotocorriente

Is: Corriente de saturación del diodo

\section{B) Tensión de circuito abierto (Voc)}

Es el máximo valor de tensión en los extremos de la célula y se da cuando no está conectada a ninguna carga (Osea l=0). El Voc incrementa logaritmicamente con el incremento de la luz del sol.

$$
V_{o c}=\frac{n K_{B} T}{q} \ln \left(\frac{I_{L}}{I_{S}}+1\right)
$$

Otros conceptos de gran importancia, que se deben mencionar en células solares es:

\section{- Eficiencia Cuántica (Quantum Efficiency(QE))}

Es la fracción de fotones incidentes que son absorbidos y convertidos en pares de electrón - hueco. Es usualmente medida en función de la longitud de onda (Fig.5).

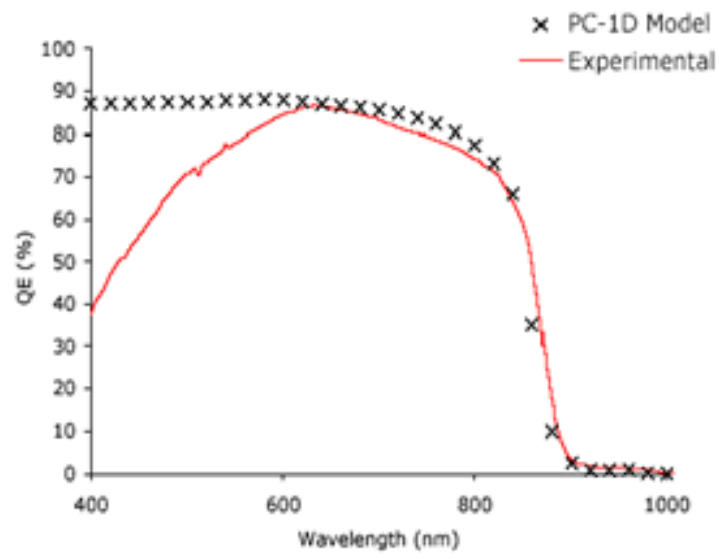

Fig.5. Respuesta de la eficiencia cuántica en función de la longitud de onda.

La eficiencia de una célula solar se define como el cociente entre la máxima potencia eléctrica que se puede entregar a la carga por la potencia incidente.

$$
\eta=\frac{P_{m}}{P_{L}} .100 \%
$$

$\mathrm{Pm}=$ Potencia máxima entregada

$\mathrm{PL}=$ Potencia incidente

\section{- Factor de Llenado}

Es la medida de la calidad de la unión y la resistencia en serie (Rs) de una célula solar. Se define como el cociente de potencia máxima que se puede entregar a una carga, entre el producto de la tensión de circuito abierto y la intensidad de cortocircuito. 


$$
F F=\frac{P_{m}}{V_{o c} * I_{s c}}
$$

El factor de forma generalmente tiene valores de $(0.7-0.8)$ para Células de GaAs.

\section{Materiales y métodos}

Mediante el programa de simulación de dispositivos semiconductores PC1D, de la Universidad de Nueva Gales del sur Australia (Hans 1993; Basore 2002) tal y como se ilustra en la Fig.6, se realiza la simulación, automáticamente los valores de las constantes son definidas y se ejecutan; el programa describe los modelos y al mismo tiempo las propiedades físicas del material y de las superficies, algunas de estas son la estructura de las bandas (Band Gap), movilidad, textura, recombinación, y las propiedades ópticas del material. Este programa permite formar células solares con un máximo de 5 regiones.

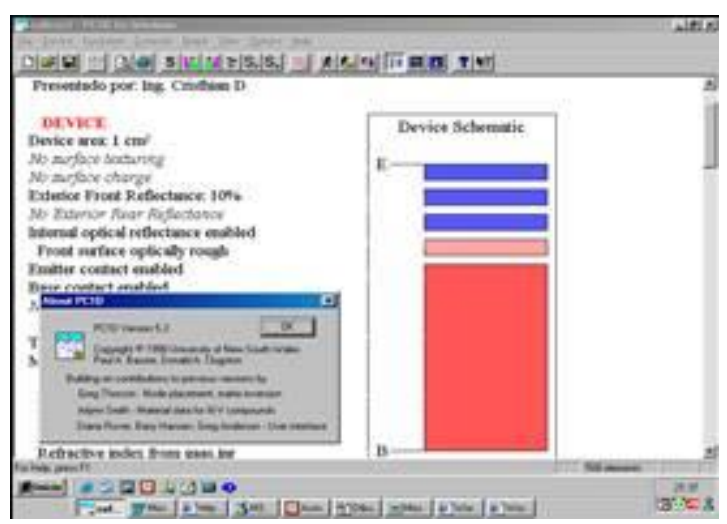

Fig.6. Programa de simulación de dispositivos semiconductores PC1D.
150

El programa de simulación nos facilita definir el tipo de material y las dimensiones del dispositivo utilizado. Para la obtención de resultados se utilizará una célula de material de GaAs con una capa fina de Al0.3Ga0.7As, con una serie de dopados Tipo $\mathrm{n}$ y tipo $\mathrm{p}$. Los valores o longitudes de la muestra y su dopado, son fijados inicialmente con sus valores estándar, pero si es necesario realizar alguna modificación es importante para comparar y analizar los resultados. La Fig.7 presenta la estructura física de la célula solar multiunión con sus respectivas regiones, en las cuales se presentan los valores de las concentraciones de dopados tipo $\mathrm{p}$ (azul) y $\mathrm{n}$ (rojo). En particular la heterounión de Al0.3Ga0.7As/GaAs. La región 4 tiene un aspecto de color menos intenso, debido a tener menos concentración de dopante, por tanto es más intrínseca que las otras. El grosor total de la célula y de cada región está también indicado.

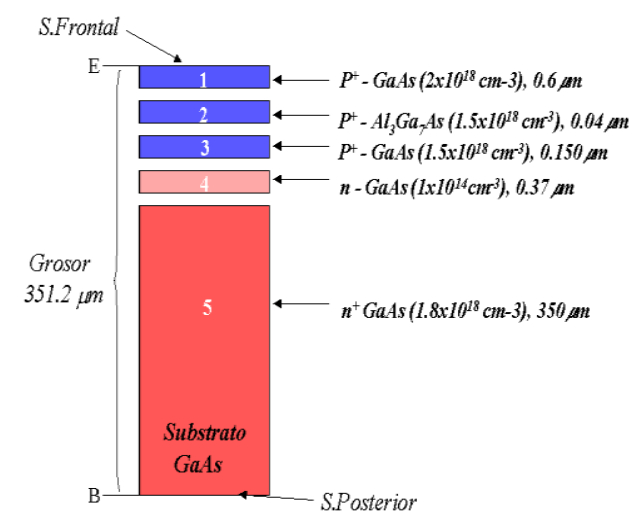

Fig.7. Estructura de la Célula Solar con 5 regiones. 
Las células multiunión son un conjunto de celdas individuales, colocadas en orden descendente de acuerdo a su espacio de banda $(\mathrm{Eg})$. La celda más alta captura los fotones de alta energía y deja pasar el resto de fotones hacia abajo para ser absorbidos por otras celdas con espacios de bandas un poco más bajos.

\section{1) Descripción del GaAs}

El GaAs, tiene una banda de separación directa y por lo tanto son posibles las transiciones verticales (Fig.8) para que los electrones puedan absorber un fotón. Estas transiciones cerca del borde de la banda de separación presentan fuerte absorción óptica. Entre las características más significativas de las Células solares con GaAs se pueden mencionar:

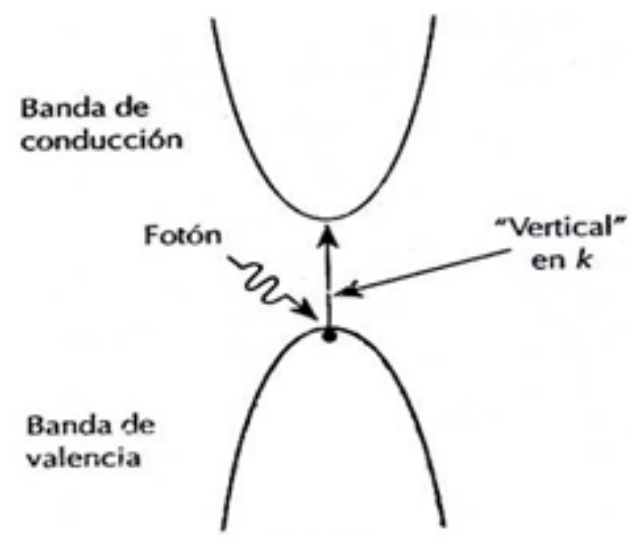

Absorción

Fig.8. Transición vertical (k) y absorción de un fotón emitiendo un electrón de una banda a otra.

La banda de separación (band gap), es de $1.43 \mathrm{eV}$.
151

Trabajan a altas temperaturas y son muy resistentes a la fuerte radiación solar.

Tiene mejor coincidencia con el espectro solar y por tanto son las más utilizadas en aplicaciones espaciales. Debido a que el programa PC1D cuenta con valores establecidos, es importante ajustarlos en forma conveniente para que los resultados obtenidos se asemejen a los experimentales 0 a los planteados teóricamente.

\section{2) Ajuste de Parámetros}

Para el GaAs, se ajustó la razón de la densidad efectiva de la banda de conducción por la de densidad efectiva de la banda de valencia:

$$
\frac{N c}{N v}=0.057
$$

> Por medio del sistema $\mathrm{Al}(\mathrm{x}) \mathrm{Ga}(1-$ $x)$ As y en el material de Al0.3Ga0.7As donde $x=0.3$, se hicieron los ajustes necesarios por las siguientes ecuaciones (Clugston 1997).

Banda de separación (Band Gap) de Al0.3Ga0.7As:

$$
E_{g}(\Gamma)=E_{g}(300)+x(1.155+0.37)
$$

Obteniéndose, $\quad E_{g}(\Gamma)=1.79 \mathrm{eV}$

Afinidad del electrón:

$$
\chi=4.07-0.85\left(E_{g}(\Gamma)-E_{g}(G a A s)\right)
$$


Bistua:Revista de la Facultad de Ciencias Básicas.2018.16(1):145-155

Resultando, $\chi=3,75 \mathrm{eV}$

Propiedades dieléctricas:

(Permitividad):

$$
\varepsilon=13.18-2.9 x
$$

Por ultimo obteniendo, $\varepsilon=12.31$

En la Fig. 9 se muestra una imagen típica de una Heteroestructura de AlGaAs/GaAs, la cual se caracteriza por una punta o cima y permite una interface entre la unión p-n.

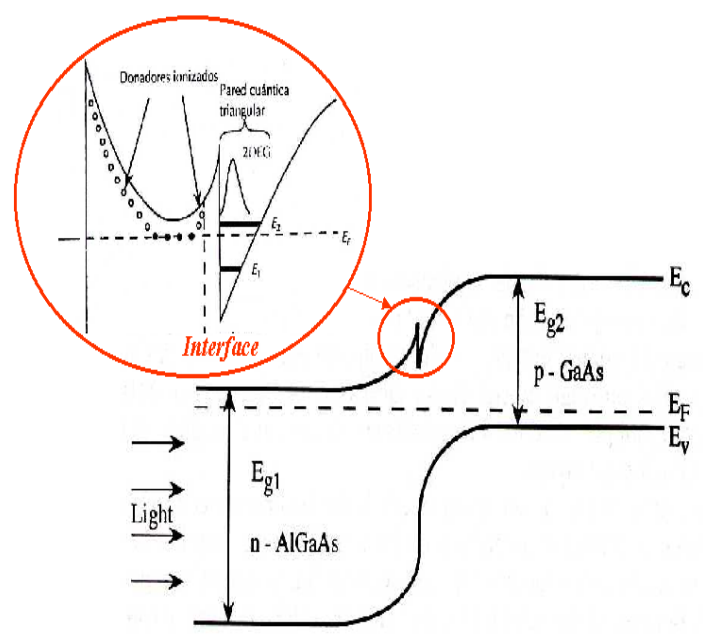

Fig.9. Heteroestructura de AIGaAs/GaAs

La imagen permite una referencia para encontrar la forma característica de la Heteroestructura de la célula solar en la simulación, a través del diagrama de bandas.

\section{Resultados y discusión}

Se encontraron los parámetros fundamentales de la célula solar por medio del programa, a través de los valores calculados en cada material. Al mismo tiempo se compararon con otros modelos obtenidos en diferentes fuentes de información.

\section{Bandas de Energía (Energy Bands):}

En la Fig. 10 se observa el diagrama de bandas de la unión $p-n$ de la célula solar. En equilibrio la distribución de electrones y huecos está dada por la distribución de fermi por lo tanto Efn = Efp. Los electrones están en equilibrio térmico en la banda de conducción y los huecos en la banda de valencia.

Si los electrones y huecos en exceso se inyectan en el semiconductor, el nivel de fermi del electrón Efn se mueven hacia la banda de conducción, mientras que el nivel de fermi del hueco Efp, lo hace a la banda de valencia (Fig. 11).

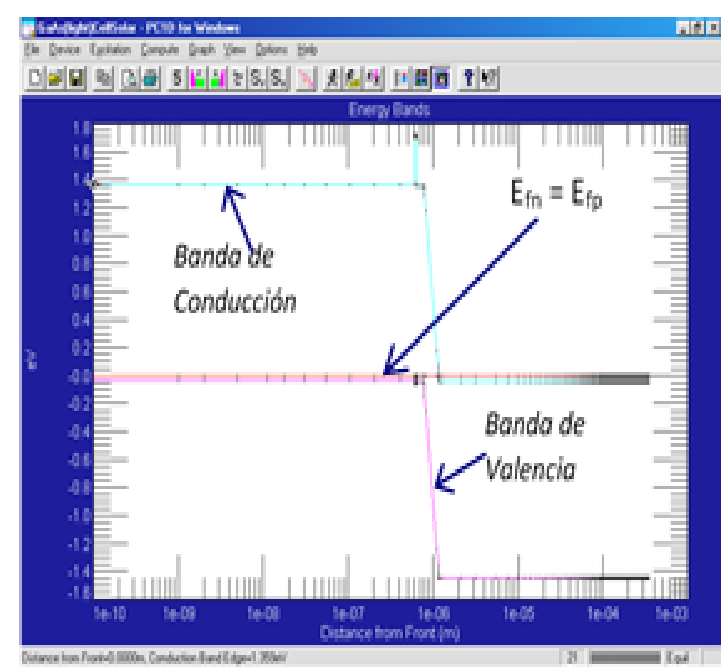

Fig.10. Diagrama de Bandas de Energía en equilibrio térmico. 


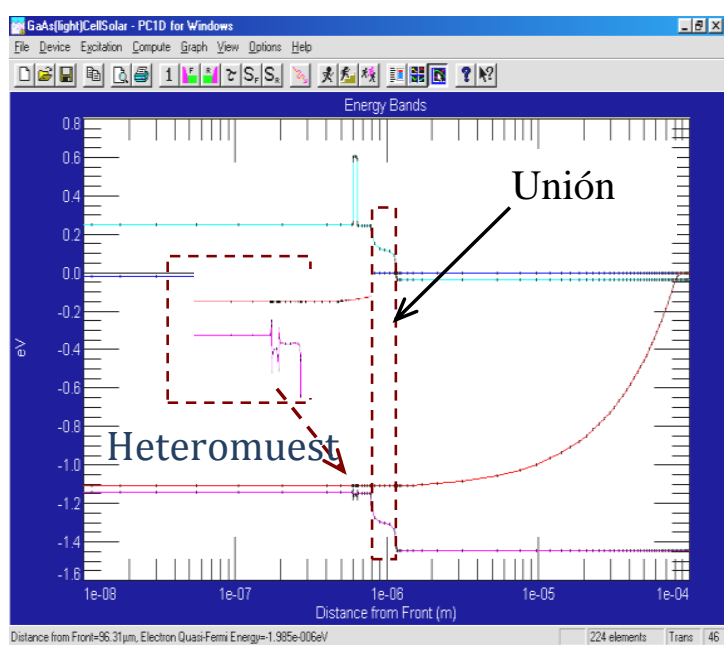

Fig.11. Separación de los niveles de energía de fermi por el exceso de portadores.

Se observa la heteromuestra de Al0.3Ga0.7As, el cual conforma la heteroestructura con el GaAs tipo p. Al mismo tiempo se ve claramente la unión p-n y la separación de los niveles de energía, debido a la tensión de Voc.

\section{Curvas de I-V y potencia Máxima}

Se presentan las curvas de I-V de base, para dos niveles de iluminación, uno bajo el espectro de radiación solar (AM 1.5), Fig.12 y luego otro con el espectro de cuerpo oscuro (Black Body) Fig.13. Estos niveles de iluminación son tomados como primera fuente y se utilizó como intensidad del espectro solar (1 Sol), el valor correspondiente a $100 \mathrm{~mW} / \mathrm{cm} 2$ Las diferencias se observan en el cambio de la intensidad de fotocorriente (IL), esta es debido al aumento de la cantidad de fotones absorbidos por la célula solar. 153

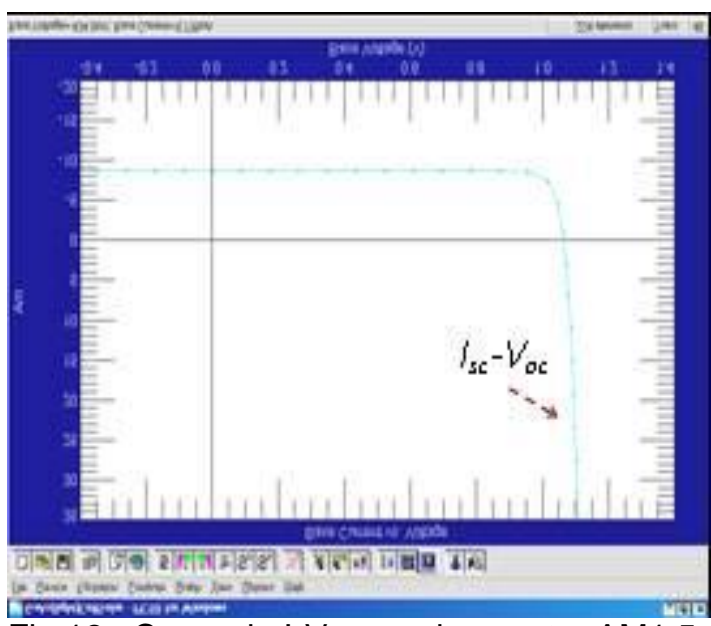

Fig.12. Curva de I-V para el espectro AM1.5.

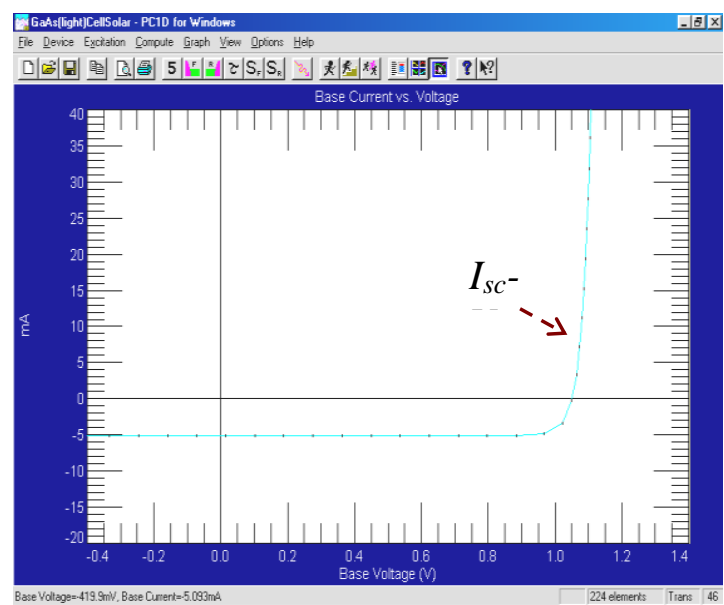

Fig.13. Curva de I-V para el espectro oscuro (Black Body).

Para calcular la potencia de la célula solar con las gráficas de I-V y bajo iluminación solar, se necesitan ubicar los dos puntos correspondientes a Isc y Voc encontrando los valores, y realizando el producto de los 2 parámetros.

El programa de simulación PC1D instantáneamente realiza esta operación y presenta la curva de potencia máxima Fig.14. 


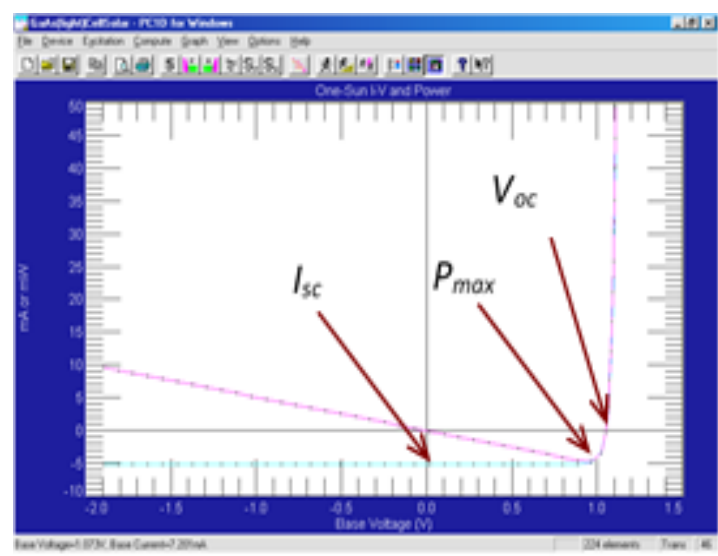

Fig.14. Curva de I-V y de potencia máxima (Pmax).

\section{Eficiencia Cuántica}

Para encontrar la curva de la eficiencia cuántica se aplicó un espectro monocromático con longitudes de onda, ajustado desde $600 \mathrm{~nm}$ a 800 $\mathrm{nm}$ ó aplicando el fichero scan-q.exe.

En la Fig. 15 se puede observar que inicialmente el comportamiento de la eficiencia de la célula solar a longitudes de onda de $600 \mathrm{~nm}-750$ nm no presenta buenos resultados, alcanza un porcentaje de $6 \%-23 \%$.

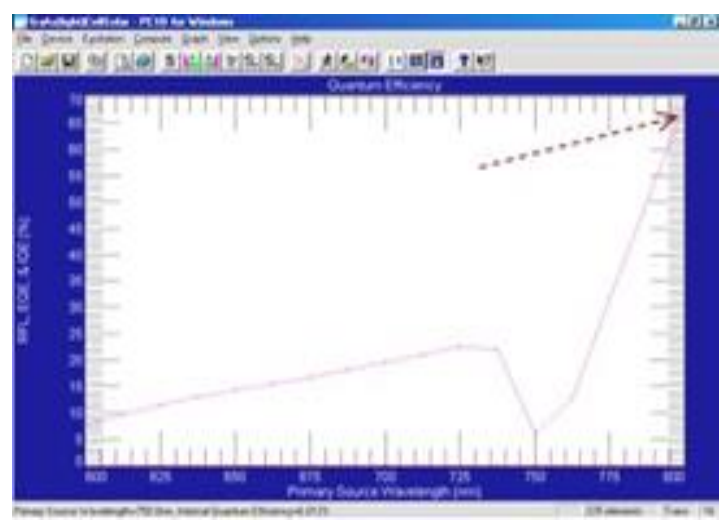

Fig.15. Curva la eficiencia cuántica con excitación monocromática.
Luego a una mayor longitud de onda de $750 \mathrm{~nm}$ dada por la fuente principal, la eficiencia aumenta en forma considerablemente y llega a $70 \%$. Este porcentaje indica el rendimiento de la célula solar.

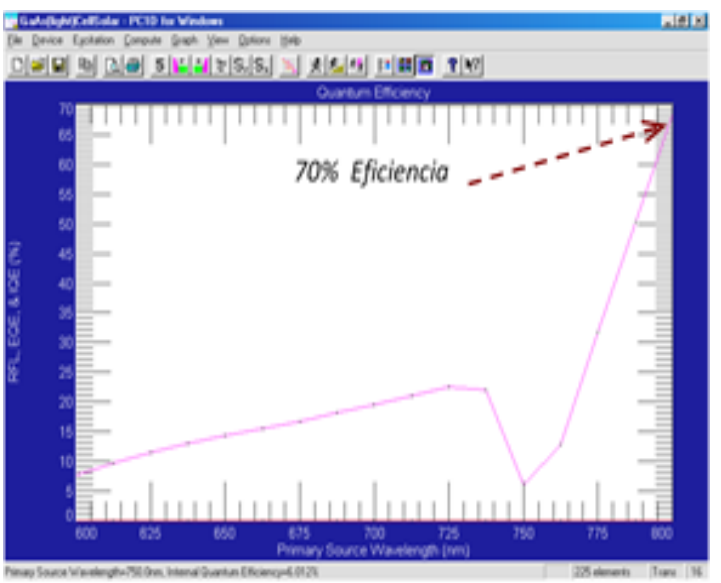

Fig.15. Curva la eficiencia cuántica con excitación monocromática.

\section{Conclusiones}

Se determinaron las respuestas de I-V para una célula solar de $\mathrm{GaAs} / \mathrm{Al0.3Ga0.7As}$ para dos espectros de iluminación.

En las células solares su efectividad se determina, a partir de la cantidad de potencia eléctrica que pueda entregar a la carga por la potencia recibida.

Las células de varias celdas son muy eficientes por que pueden convertir una fracción más grande del espectro luminoso en electricidad.

Conociendo los parámetros del material semiconductor y las diferentes maneras de restar las 
155

perdidas ópticas, se puede optimizar y mejorar el rendimiento del dispositivo.

El programa de simulación PC1D, es una herramienta muy efectiva y apropiada para la simulación de dispositivos semiconductores.

\section{Referencias Bibliográficas}

Hauga P, Birger R. Olaisena,b, rnulf Nordsetha Erik S. Marsteina,c, 2013, A graphical user interface for multivariable analysis of silicon solar cells using scripted PC1D simulations, Energy Procedia, (38): 72 -79 .

Cai, X, Zhou X, Liu Z, Jiang F, Qingchun Y, 2018, An in-depth analysis of the silicon solar cell key parameters' optimal magnitudes using PC1D simulations, Optik, (164): 2018, 105113.

Jiang C, Ze L, Zhang X, Hou L, 2011, Simulation of Silicon Solar Cell Using PC1D, Advanced Materials Research, (383-390): 7032-7036.

Molina M, Tebaldi M, Bolognini N..2013.Eficiencia de difracción del registro de speckle modulados generados a partir de superficies reflecto-difusoras. Bistua:Revista de la Facultad de Ciencias Basicas. 10 (2):5060.

Hussain B, Ebong A, 2015, Specifications of $\mathrm{ZnO}$ growth for heterostructure solar cell and PC1D based simulations, Open access, Data in Brief, (5): 516-521.

Singh J. Semiconductor Devices, Basic Principles, 2001, John Wiley \& Sons, Inc. 455517.
Liou J, 1994, Advanced Semiconductor Device Physics and Modeling, First Edition edition 407-449.

Wenham S, Green M, Murie Watt E, 2011, Applied Photovoltaics, Centre for photovoltaic Devices and System. 290.

Hans M, Semiconductors for solar cells, Artech House.Inc, 1993.

Basore P.A, Clugston D.A, 2002, PC1D for Windows: From Analysis to Design', 25th IEEE PVSC, 377-381.

Clugston D. A, Basore P.A, 1997, Modelling Freecarrier Absorption in Solar Cells', Progress in Photovoltaics, (5)4: 229-236.

*Para citar este artículo:Duran Acevedo CM. Optimization of a solar Cell of GaAs/AlGaAs.Revista.Bistua.2018.16(1):145 $-155$

+ Autor para el envio de correspondencia y la solicitud de las separatas: Cristhian Manuel Durán Acevedo. Departamento de Electrónica, Eléctrica, Sistemas y Telecomunicaciones .Facultad de Ingenierías y Arquitectura. Universidad de Pamplona., Colombia. cmduran@unipamplona.edu.co

Recibido: Enero 22 de 2017

Aceptado: Febrero 10 de 2018 